\title{
Prestenting Versus Nonprestenting on the Outcomes of Flexible Ureteroscopy for Large Upper Urinary Stones: A Systematic Review and Meta-Analysis
}

\author{
Xueliang Chang Yaxuan Wang Jingdong Li Zhenwei Han \\ Department of Urology, Second Hospital of Hebei Medical University, Shijiazhuang, China
}

\section{Keywords}

Prestenting · Flexible ureteroscopy · Upper urinary stone ·

Meta-analysis

\begin{abstract}
Introduction: The purpose of this article is to evaluate the efficacy and safety of prestenting (PS) versus non-PS (NPS) of flexible ureteroscopy (fURS) to treat large upper urinary stones. Methods: We conducted a systematic literature research of PubMed, Ovid, Scopus (up to August 2019), and citation lists to identify eligible studies. All studies comparing PS versus NPS of fURS were included. Data were analyzed using the Cochrane Collaboration's Review Manager (RevMan) 5.3 software. Results: Overall, 7 studies including 3,145 patients (PS 1,408; NPS 1,737) were included in this article. PS group was associated with older age (weighted mean difference [WMD] 0.91 year; $p<0.001$ ) and more male patients (odds ratio $[O R] 1.34 ; p<0.001$ ). There were no statistical differences between PS and NPS in BMI (WMD $0.34 \mathrm{~kg} / \mathrm{m}^{2} ; p=$ $0.13)$, stone size (WMD $0.13 \mathrm{~mm} ; p=0.77$ ), and operative time (WMD 0.44 min; $p=0.86$ ). Compared with NPS, PS showed better initial success rate (OR 4.04; $p<0.001)$ and higher SFR (OR 1.64; $p<0.001)$. There were no statistical differences for complications (OR 0.84; $p=0.42$ ) and Clavien-
\end{abstract}

Dindo score $\geq 3$ complications (OR 1.04; $p=0.93$ ). Conclusion: $P S$ could improve initial success rate and avoid secondary general anesthesia for first ureteral access sheath failed patients. PS could provide better SFR than NPS in the treatment of large upper urinary stones with fURS.

(c) 2021 S. Karger AG, Basel

\section{Introduction}

With the improvement of surgical instruments and techniques, flexible ureteroscopy (fURS) has been increasingly used in the treatment of upper urinary stones [1]. The EAU guidelines recommend fURS as an effective and safe option for upper urinary stone $<2 \mathrm{~cm}$ [2]. Due to the characteristics of fURS and the anatomy of ureter, fURS usually requires the assistance of a ureteral access sheath (UAS). Stern et al. [3] reported that UAS could help fURS easily and repeatedly pass through the ureter. In addition, UAS could reduce the incidence of postoperative urinary tract infections by maintaining a lower intrarenal pressure [4]. However, Traxer and Thomas [5] reported that UAS may cause ureteral wall damage. Therefore, many studies have introduced double-J stents before fURS in order to improve SFR and reduce ureteral
Correspondence to:

Zhenwei Han, hanzhenwei1101@126.com 
Fig. 1. PRISMA flow diagram of the search strategy and identification of studies included in this study.

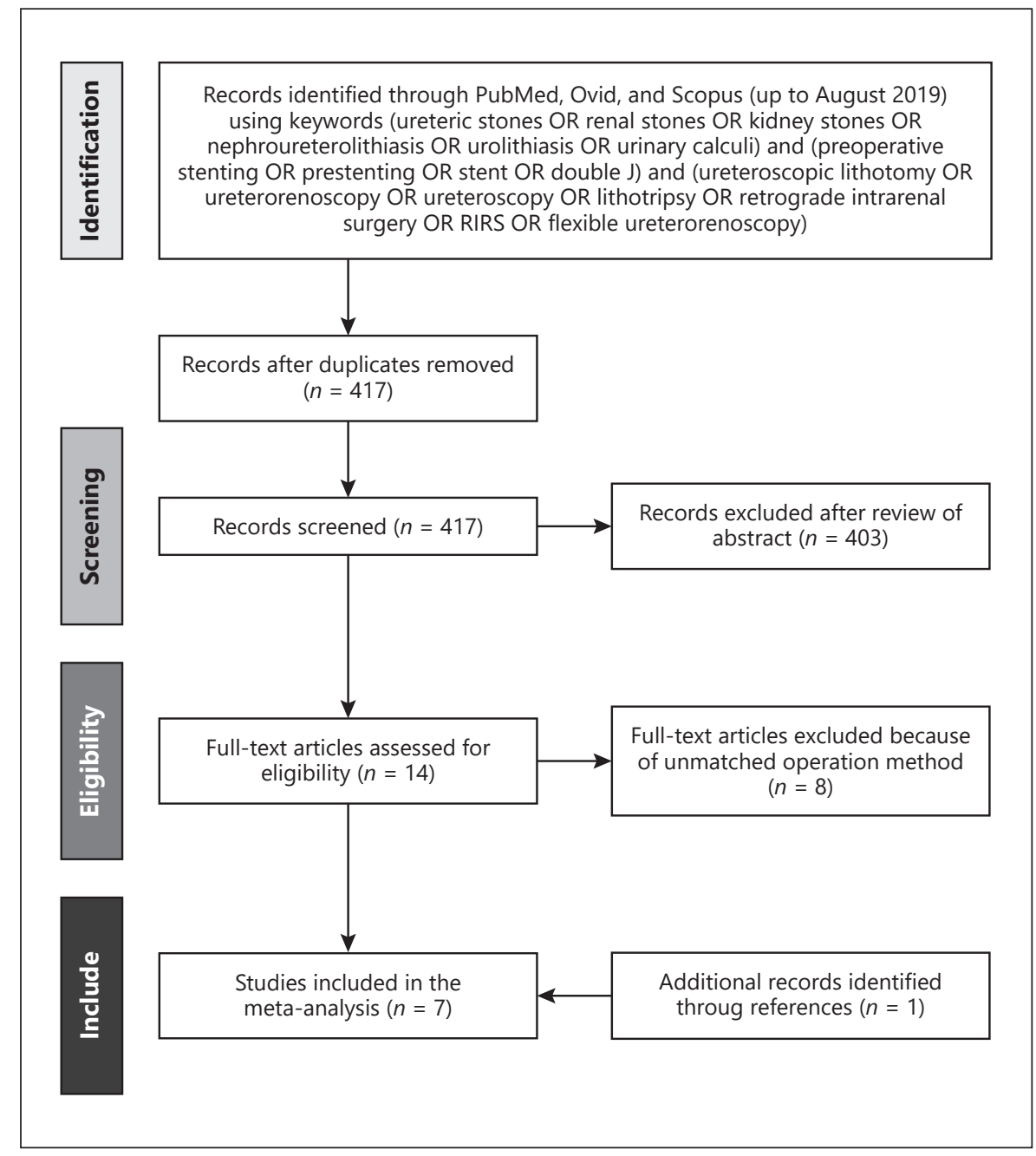

injuries. However, prestenting (PS) of fURS has not been recommended as a routine procedure by guidelines [2].

In the treatment of large upper urinary stones, whether PS is superior to non-PS (NPS) remains controversial. Compared with NPS, PS will cost more and bring additional complications, such as fever, lower urinary tract symptoms, and hematuria [6]. If PS is not significantly better than NPS in terms of surgical success rate, SFR, and complications, PS should not be used routinely. The purpose of this article is to perform a meta-analysis to evaluate the efficacy and safety of PS versus NPS of fURS to treat large upper urinary stones.

\section{Methods}

Search Strategy

Articles comparing PS versus NPS of fURS were systematically searched in PubMed, Ovid, and Scopus (up to August 2019). The search strategy was "(ureteric stones OR renal stones OR kidney stones OR nephroureterolithiasis OR urolithiasis OR urinary calculi) and (preoperative stenting OR prestenting OR stent OR double $J$ ) and (ureteroscopic lithotomy OR ureterorenoscopy OR ureteroscopy OR lithotripsy OR retrograde intrarenal surgery OR RIRS OR flexible ureterorenoscopy)." The titles and abstracts of potentially eligible studies were screened by 2 investigators (X.C. and Y.W.) independently. Relevant citations of retrieved studies were also checked for additional eligible studies.

\section{Inclusion and Exclusion Criteria}

Inclusion criteria: [1] the study included adult patients only and written in the English language; [2] the stone size was $\geq 10 \mathrm{~mm}$; [3] patients were treated with fURS; and [4] the outcomes compared PS and NPS. Exclusion criteria: [1] children studies; [2] the stone size was $<10 \mathrm{~mm}$; and [3] studies without primary data (i.e., reviews, commentaries, and conference abstracts).

\section{Data Extraction}

Two researchers (X.C. and Y.W.) independently reviewed the potential eligible titles and abstracts. Then the full texts of qualified 
Table 1. Basic characteristics of included studies

\begin{tabular}{|c|c|c|c|c|c|c|c|c|c|c|}
\hline Study & Period & $\begin{array}{l}\text { Study } \\
\text { design }\end{array}$ & Region & $\begin{array}{l}\text { Stone location } \\
\text { of PS }(\mathrm{K} / \mathrm{U} / \mathrm{M})\end{array}$ & $\begin{array}{l}\text { Stone location of } \\
\text { NPS }(\mathrm{K} / \mathrm{U} / \mathrm{M})\end{array}$ & PS time & $\begin{array}{l}\text { Definition } \\
\text { of SFR }\end{array}$ & $\begin{array}{l}\text { Follow-up } \\
\text { methods }\end{array}$ & $\begin{array}{l}\text { Follow-up } \\
\text { time }\end{array}$ & LE \\
\hline Assimos et al. [8] & $2010-2012$ & PRP & Global & $590 /-/-$ & $1,002 /-/-$ & ns & $<1 \mathrm{~mm}$ & ns & ns & 4 \\
\hline Chen et al. [9] & $2015-2017$ & RTP & China & $43 / 25 / 31$ & $43 / 39 / 18$ & $1-2$ weeks & $<4 \mathrm{~mm}$ & KUB/CT & 3 months & 4 \\
\hline Dessyn et al. [10] & 2004-2010 & RTP & France & $238 / 96 / 70$ & $158 / 30 / 48$ & ns & Complete & $\begin{array}{l}\mathrm{CT} / \mathrm{KUB}+ \\
\mathrm{US}\end{array}$ & 6 months & 4 \\
\hline Kawahara et al. [12] & $2010-2011$ & RTP & Japan & $25 /-/-$ & $36 /-/-$ & $1-2$ weeks & $<4 \mathrm{~mm}$ & $\mathrm{KUB}+\mathrm{CT}$ & 6 weeks & 4 \\
\hline Lee et al. [13] & 2011-2017 & RTP & South Korea & $168 /-/-$ & $215 /-/-$ & $\geq 7$ days & $\leq 1 \mathrm{~mm}$ & $\mathrm{KUB} / \mathrm{CT}$ & $1-4$ weeks & 4 \\
\hline Zhang et al. [14] & 2013-2015 & RTP & China & $56 /-/-$ & $100 /-/-$ & ns & $\leq 4 \mathrm{~mm}$ & KUB/CT & 3 months & 4 \\
\hline
\end{tabular}

PS, prestenting; K, kidney; U, ureter; M, multiple; SFR, stone-free rate; PRP, prospective; RTP, retrospective; ns, not specified; KUB, kidney ureter bladder X-ray film; CT, computed tomography; US, ultrasound; ES, endoscopy; LE, level of evidence according to the Oxford Centre for Evidence-based Medicine.

articles were examined for further consideration. Data were extracted by using a predefined data extraction form, including demographic characteristics (age, gender, BMI, and stone size) and surgical outcomes (operative time, initial success rate, stone-free rate, and complications). Any disagreement was resolved in consultation with J.L.

\section{Quality Assessment}

Two researchers (X.C. and Y.W.) independently assessed the evidence level of included studies according to the Oxford Centre for Evidence-based Medicine. Discrepancy was resolved in consultation with the third researcher (J.L.).

\section{Statistical Analysis}

A meta-analysis was conducted using Cochrane Collaboration's Review Manager (RevMan) 5.3 software (Cochrane Collaboration, Oxford, UK). For continuous data, the weighted mean difference (WMD) with 95\% confidence intervals (CIs) was used. Otherwise, odds ratio (OR) with 95\% CI was calculated. Because only means and standard deviations are permitted for continuous data in RevMan 5.3, a validated mathematical model was used to convert median (range) to mean (standard deviation) for studies only reporting median and range [7]. Statistical heterogeneity was defined based on the Cochrane $Q p$ value or $I^{2}$ statistic. If $p>0.1$ or $I^{2}<50 \%$, a fixedeffects model was used. While, if $p<0.1$ or $I^{2}>50 \%$, a random-effects model was used. A $p$ value $<0.05$ was considered statistically significant. Funnel plots were examined to evaluate publication bias.

\section{Results}

Overall, 7 studies including 3,145 patients (PS 1,408; NPS 1,737) were included in this article (Fig. 1) [8-14]. Table 1 shows the basic characteristics and quality assessment of the included studies.
Six studies including 2,888 patients (PS 1,254; NPS $1,634)$ that met the inclusion criteria reported age and gender [8-10, 12-14]. The PS group was found to be associated with older age (WMD 0.91 year, $95 \%$ CI $0.72-$ $1.09 ; p<0.001$ ) and more male patients (OR 1.34, 95\% CI $1.14-1.56 ; p<0.001)$. Jessen et al. [11] reported upper urinary stones $<10 \mathrm{~mm}$, we could not get the age and gender data for stones $\geq 10 \mathrm{~mm}$. Four studies including 2,446 patients (PS 1,028; NPS 1,418) reported BMI $[8,10,13$, 14]. The results showed no statistically significant difference between PS and NPS (WMD $0.34 \mathrm{~kg} / \mathrm{m}^{2}, 95 \%$ CI -0.09 to $0.77 ; p=0.13$ ). Six studies including 1,549 patients (PS 814; NPS 735) reported stone size [9-14]. It seemed that PS was associated with smaller stone size (WMD $-0.10 \mathrm{~mm}, 95 \% \mathrm{CI}-0.17$ to $-0.03 ; p=0.005$ ) (Fig. 2). However, we found that Dessyn et al. [10] provided about $99.4 \%$ weight, which makes other studies not meaningful. And Dessyn et al. [10] reported similar stone sizes between the 2 groups. Therefore, we assessed the stone size after omitting the data of this study. The result showed no statistical significance between PS and NPS (WMD $0.13 \mathrm{~mm}, 95 \% \mathrm{CI}-0.74$ to $1.01 ; p=0.77$ ). (see online suppl. Fig. 1; for all online suppl. material, see www. karger.com/doi/10.1159/000506652).

Seven studies including 3,094 patients (PS 1,396; NPS $1,698)$ reported operative time [8-14]. The results showed no statistical difference between PS and NPS (WMD 0.44 $\min , 95 \% \mathrm{CI}-4.26$ to $5.14 ; p=0.86$ ). Three studies including 436 patients (PS 186; NPS 250) reported initial success rate $[9,12,14]$. The meta-analysis demonstrated a significantly higher initial success rate in PS than NPS (OR 


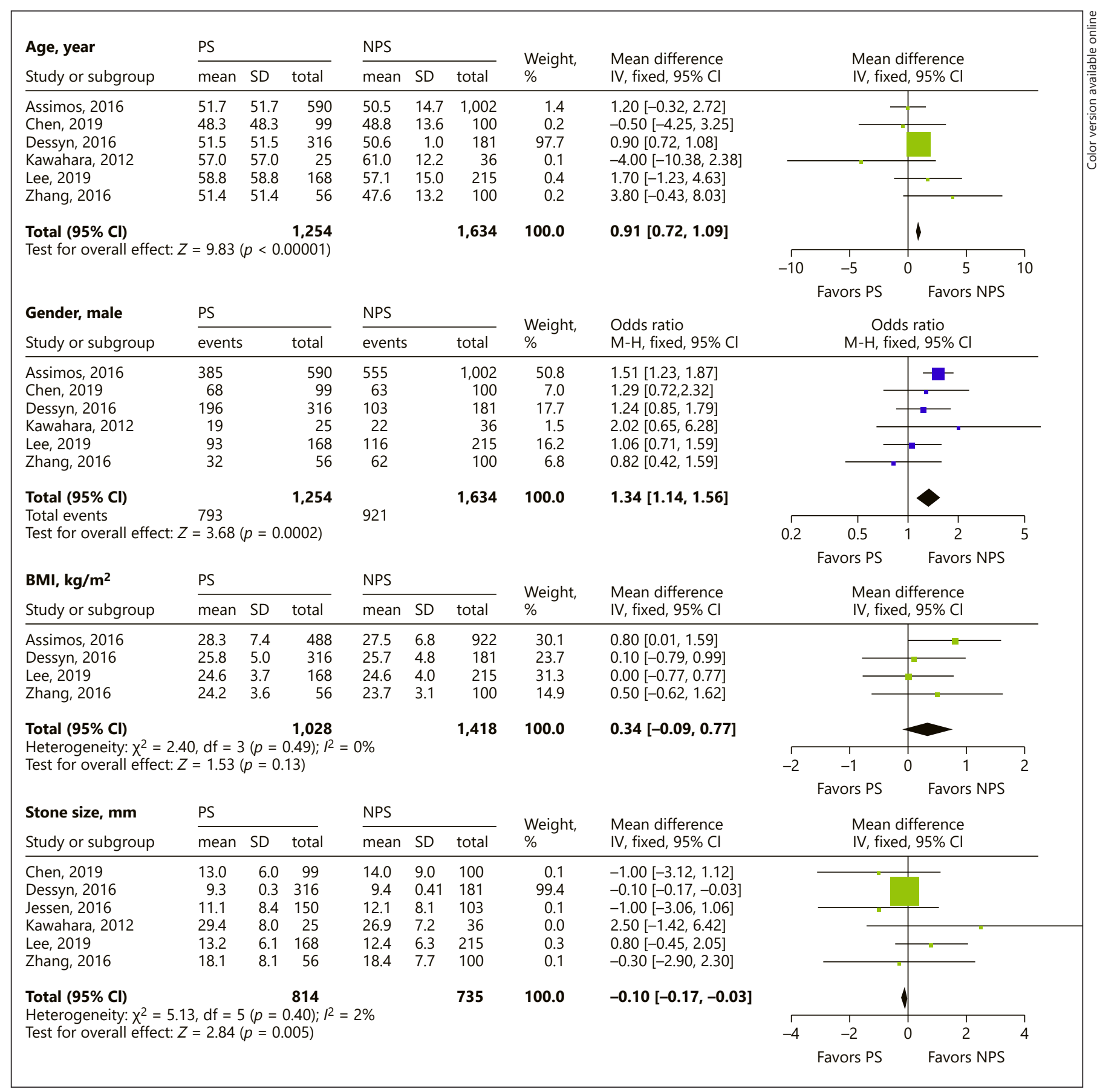

Fig. 2. Forest plots of demographic characteristics of PS versus NPS of fURS for upper urinary stones. SD, standard deviation; CI, confidence interval; PS, prestenting; NPS, non-PS; fURS, flexible ureteroscopy.

4.04, 95\% CI 1.87-8.73; $p<0.001)$. Seven studies including 3,097 patients (PS 1,407; NPS 1,690) reported stonefree rate [8-14]. Meta-analysis of these studies showed that PS had higher SFR than NPS (OR 1.64, 95\% CI 1.23$2.18 ; p<0.001$ ). (Fig. 3).

Prestenting for Large Upper Urinary Stones
Seven studies including 3,138 patients (PS 1,414; NPS 1,724) reported complications [8-14]. Five studies including 1,291 patients (PS 663; NPS 628) reported complications that could be assessed with Clavien-Dindo score system $[9,10,12-14]$. The results showed no statis-

Urol Int 2021;105:560-567 


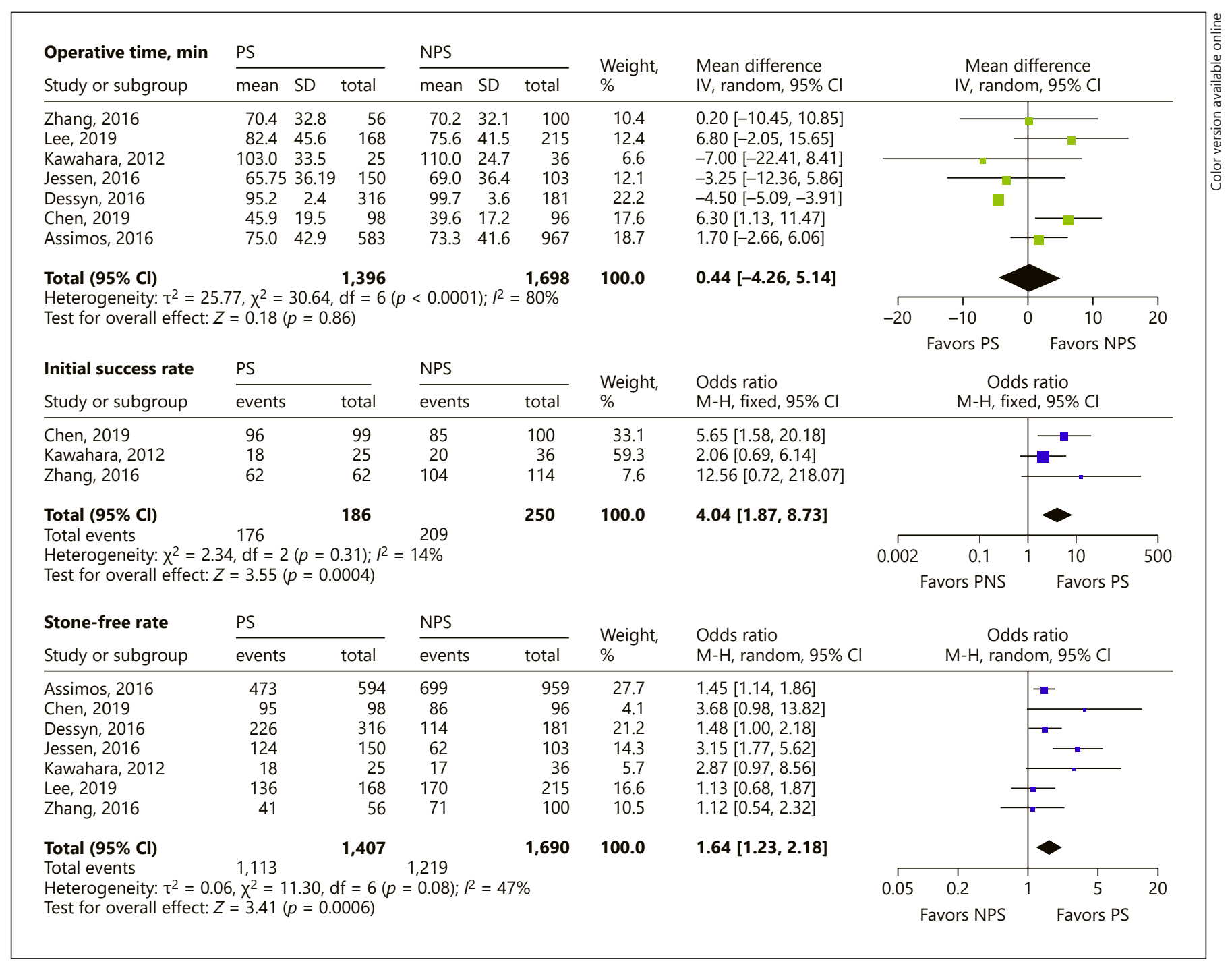

Fig. 3. Forest plots of surgical outcomes of PS versus NPS of fURS for upper urinary stones. SD, standard deviation; CI, confidence interval; PS, prestenting; NPS, non-PS; fURS, flexible ureteroscopy.

tically significant difference for complications (OR 0.84 , 95\% CI 0.54-1.29; $p=0.42$ ) and Clavien-Dindo score $\geq 3$ complications (OR 1.04, 95\% CI $0.42-2.61 ; p=0.93$ ) (Fig. 4). There was no significant publication bias in this study. The funnel plots of this meta-analysis were summarized in online suppl Fig. 2.

\section{Discussion}

fURS has been increasingly used for proximal ureteral and renal stones, which is considered a first-line treatment of upper urinary stones $<2 \mathrm{~cm}[1]$. UAS has been reported to improve surgical outcomes and reduce complications, and UAS combined with fURS is recommended as a good option by EAU guidelines [2]. The initial failure rate of UAS was reported about $4-10 \%[9,14]$. However, it has been reported that PS of fURS had a higher insertion rate of UAS $[9,15,16]$. Kawahara et al. [12] reported the success rate of 14/16Fr UAS for PS and NPS groups. They found that PS was associated with higher success rate $(94.7 \%, 18 / 19)$ than NPS $(74.2 \%, 23 / 31)$. In addition, some studies reported PS was associated with higher SFR and less complications [5, 17, 18]. Meanwhile, other studies reported that PS could lead to extra cost and complications without superior benefits $[6,14,15,19$, 


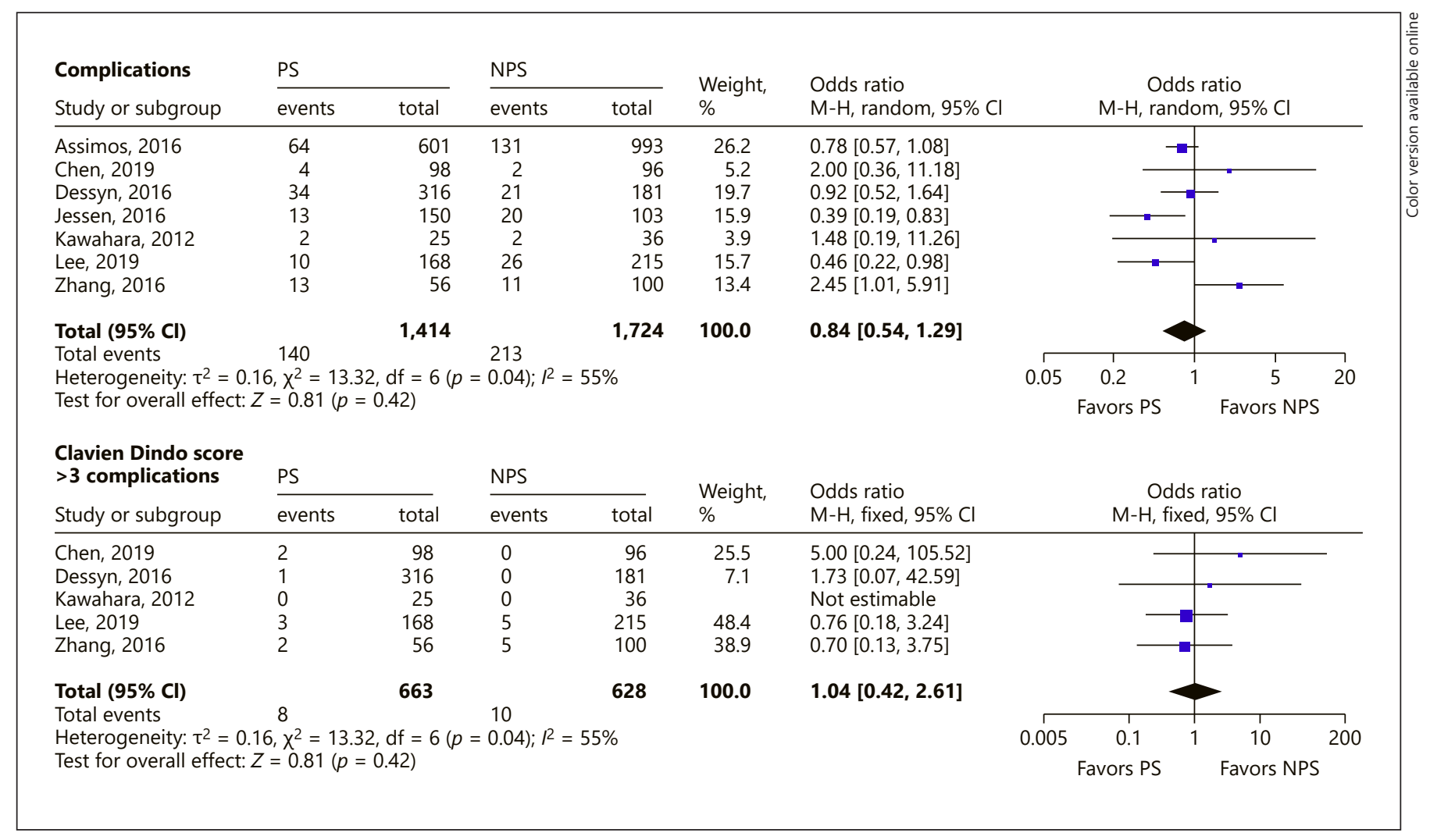

Fig. 4. Forest plots of complications of PS versus NPS of fURS for upper urinary stones. CI, confidence interval; PS, prestenting; NPS, non-PS; fURS, flexible ureteroscopy.

20]. In this meta-analysis, we attempted to assess the efficacy and safety of PS compared with NPS of fURS to treat large upper urinary stones.

In fURS, some studies have demonstrated that PS would passively dilate ureter, and the dilated ureter would make it easier to place UAS. In this meta-analysis, we found that the initial success rate was higher in PS group. Although the use of UAS could not be extracted, we believe that PS could improve the success rate of UAS or that PS could improve the ureter condition and facilitate the entry of ureteroscopy. As described by Chen et al. [9], PS could improve the ureter condition, which would be a very good opportunity for amateur surgeons. Otherwise, PS is an easy procedure and could be done within $10 \mathrm{~min}$ in outpatient ward. PS could avoid secondary general anesthesia for first UAS failed patients [9].

With the help of UAS or the dilated ureter alone will shorten the operative time $[9,12,13]$. So, the surgeon can break more stones in a limited time. Rubenstein et al. [18] reported that PS could improve SFR significantly for rigid and/or flexible ureteroscopy $(p=0.048)$. Assimos et al. [8] reported that PS increased SFR and decreased complications in patients with renal stones but not in those with ureteric stones. Jessen et al. [11] found that PS could improve SFR and reduce complication rate for renal stones. There was no significant difference in SFR of ureteral stones. However, the complication rate of NPS was significantly higher. However, the impact of PS on SFR of upper urinary stones remains controversial. Zhang et al. [14] reported no significant differences in SFR between PS and NPS groups $(p=0.854)$. Lee et al. [13] found that PS had no significant effect on SFR $(p=$ 0.433 ). In this meta-analysis, we found that the SFR of large upper urinary stones in PS group was significantly better. This result is consistent with most of the recent studies. The reasons might be as follows: [1] PS straightened and dilated ureter, making it easier to access and remove stones. [2] Better irrigation and drainage led to clearer vision. [3] A relatively longer effective operative time allowed surgeons to break more stones. [4] Residual stones could be more easily expelled through the dilated ureter. 
In our meta-analysis, we found that patients with PS were older, which could also reflect the complexity of patients with PS. The reasons of PS were not able to stratify, most of which were to relieve complications such as renal colic, obstruction, and urinary sepsis. Although the condition of PS patients was more complicated, the initial success rate and SFR were still better than those of NPS patients. Therefore, it can better reflect the effectiveness of PS.

Obviously, PS is an extra procedure that will lead to extra costs and various complications, including flank pain, hematuria, and lower urinary tract symptoms [19]. Many studies evaluated the complications of PS versus NPS. Most of them reported that complications were not significantly different between PS and NPS. However, Lee et al. [13] found that PS could prevent high-grade ureteral injuries and reduce the rate of intraoperative balloon dilation. Jessen et al. [11] found that PS could reduce complication rate for both renal stones and ureteral stones. In our meta-analysis, we assessed the overall complications and Clavien-Dindo score $\geq 3$ complications. There were no statistically significant differences between PS and NPS in the above 2 aspects.

We acknowledge the fact that there are a number of limitations in our study. First, most of the studies included in our meta-analysis were retrospective. The retrospective nature limited the quality of the results. Second, the use of UAS was not clear and the types of UAS were not consistent. This might lead to selection bias. Third, the surgical techniques could not be uniformed. Fourth, the stone locations were not consistent which might lead to heterogeneity. Fifth, the duration of PS was not consistent. Finally, the follow-up time was short and we were unable to assess long-term complications such as ureteral stricture.

\section{Conclusion}

Although PS is an additional procedure with some complications, it could improve initial success rate and avoid secondary general anesthesia for first UAS failed patients. PS could provide better SFR than NPS in the treatment of large upper urinary stones with fURS. This meta-analysis could provide some valuable information in decision-making for urologists and patients.

\section{Acknowledgements}

We thank Dr. Chawnshang Chang at University of Rochester Medical Center for helping with the preparation of the manuscript.

\section{Statement of Ethics}

No approval is required.

\section{Conflict of Interest Statement}

The authors have no conflicts of interest to declare.

\section{Funding Sources}

This research did not receive any specific grant from funding agencies in the public, commercial, or not-for-profit sectors.

\section{Author Contributions}

All authors contributed to the study conception and design. Material preparation, data collection, and analysis were performed by Xueliang Chang, Yaxuan Wang, and Jingdong Li. The first draft of the manuscript was written by Zhenwei Han, and all authors commented on previous versions of the manuscript. All authors read and approved the final manuscript.

\section{References}

1 Pietropaolo A, Proietti S, Geraghty R, Skolarikos A, Papatsoris A, Liatsikos E, et al. Trends of "urolithiasis: interventions, simulation, and laser technology" over the last 16 years (2000-2015) as published in the literature (PubMed): a systematic review from European section of uro-technology (ESUT). World J Urol. 2017;35(11):1651-8.

2 Turk C, Petrik A, Sarica K, Seitz C, Skolarikos $\mathrm{A}$, Straub M, et al. EAU guidelines on interventional treatment for urolithiasis. Eur Urol. 2016;69(3):475-82.

3 Stern JM, Yiee J, Park S. Safety and efficacy of ureteral access sheaths. J Endourol. 2007; 21(2):119-23.
4 Auge BK, Pietrow PK, Lallas CD, Raj GV, Santa-Cruz RW, Preminger GM. Ureteral access sheath provides protection against elevated renal pressures during routine flexible ureteroscopic stone manipulation. J Endourol. 2004;18(1):33-6.

5 Traxer O, Thomas A. Prospective evaluation and classification of ureteral wall injuries resulting from insertion of a ureteral access sheath during retrograde intrarenal surgery. J Urol. 2013;189(2):580-4.
6 Al-Marhoon MS, Shareef O, Venkiteswaran $\mathrm{KP}$. Complications and outcomes of JJ stenting of the ureter in urological practice: a single-centre experience. Arab J Urol. 2012;10(4):372-7.

7 Hozo SP, Djulbegovic B, Hozo I. Estimating the mean and variance from the median, range, and the size of a sample. BMC Med Res Methodol. 2005;5:13.

8 Assimos D, Crisci A, Culkin D, Xue W, Roelofs A, Duvdevani M, et al. Preoperative JJ stent placement in ureteric and renal stone treatment: results from the Clinical Research Office of Endourological Society (CROES) ureteroscopy (URS) global study. BJU Int. 2016;117(4):648-54. 
9 Chen H, Chen G, Zhu Y, Yang Z, Xiong C, Pan Y. Analysis of prestenting on outcomes of flexible ureteroscopy for upper urinary urolithiasis: a historical control study. Urol Int. 2019;102(2):175-80.

10 Dessyn JF, Balssa L, Chabannes E, Jacquemet B, Bernardini S, Bittard H, et al. Flexible ureterorenoscopy for renal and proximal ureteral stone in patients with previous ureteral stenting: impact on stone-free rate and morbidity. J Endourol. 2016;30(10):1084-8.

11 Jessen JP, Breda A, Brehmer M, Liatsikos EN, Millan Rodriguez F, Osther PJ, et al. International collaboration in endourology: multicenter evaluation of prestenting for ureterorenoscopy. J Endourol. 2016;30(3):268-73.

12 Kawahara $\mathrm{T}$, Ito $\mathrm{H}$, Terao $\mathrm{H}$, Ishigaki $\mathrm{H}$, Ogawa $\mathrm{T}$, Uemura $\mathrm{H}$, et al. Preoperative stenting for ureteroscopic lithotripsy for a large renal stone. Int J Urol. 2012;19(9):881-5.
13 Lee MH, Lee IJ, Kim TJ, Lee SC, Jeong CW, Hong SK, et al. The effect of short-term preoperative ureteral stenting on the outcomes of retrograde intrarenal surgery for renal stones. World J Urol. 2019;37(7):1435-40.

14 Zhang J, Xu C, He D, Lu Y, Hu H, Qin B, et al. Flexible ureteroscopy for renal stone without preoperative ureteral stenting shows good prognosis. PeerJ. 2016;4:e2728.

15 Netsch C, Knipper S, Bach T, Herrmann TR, Gross AJ. Impact of preoperative ureteral stenting on stone-free rates of ureteroscopy for nephroureterolithiasis: a matched-paired analysis of 286 patients. Urology. 2012;80(6): 1214-9.

16 Chu L, Sternberg KM, Averch TD. Preoperative stenting decreases operative time and reoperative rates of ureteroscopy. J Endourol. 2011;25(5):751-4.
17 Perlmutter AE, Talug C, Tarry WF, Zaslau S, Mohseni H, Kandzari SJ. Impact of stone location on success rates of endoscopic lithotripsy for nephrolithiasis. Urology. 2008; 71(2):214-7.

18 Rubenstein RA, Zhao LC, Loeb S, Shore DM, Nadler RB. Prestenting improves ureteroscopic stone-free rates. J Endourol. 2007; 21(11):1277-80.

19 Joshi HB, Stainthorpe A, MacDonagh RP, Keeley FX Jr, Timoney AG, Barry MJ. Indwelling ureteral stents: evaluation of symptoms, quality of life and utility. J Urol. 2003; 169(3):1065-9;

20 Lumma PP, Schneider P, Strauss A, Plothe $\mathrm{KD}$, Thelen P, Ringert RH, et al. Impact of ureteral stenting prior to ureterorenoscopy on stone-free rates and complications. World J Urol. 2013;31(4):855-9. 